\title{
Sequences of measurable functions
}

by

\section{Elỉbieta Wagner (Lódź)}

Abstract. The paper consists of three parts. In the first part we shall define convergence with respect to the $\sigma$-ideal $\mathfrak{I}$ and we shall prove the necessary and sufficient condition under which this convergence yields a topology in the set of real measurable functions. The second part contains the generalization of the theorem of Goffman and Waterman ([2]) concerning upper and lower limits onto the case of convergence with respect to the $\sigma$-ideal. The third part deals with double sequences of measurable functions and includes, among other matters a generalization of the theorem of Sierpiński ([4]).

Let $(X, \mathscr{P})$ be a measurable space. Let $\mathscr{I} \subset \mathscr{P}$ be a proper $\sigma$-ideal in a $\sigma$-field $\mathscr{S}$. We shall say that $\mathscr{I}$-almost every point of $A \subset X$ has some property (or that this property holds $\mathscr{I}$-almost everywhere, in abbreviation $\mathscr{I}$-a.e., on $A$ ) if and only if the set of points in $A$ which do not have this property belongs to the $\sigma$-ideal $\mathscr{I}$.

DEFINITION 1. We shall say that the sequence $\left\{f_{n}\right\}_{n \in N}$ of $\mathscr{S}$-measurable functions defined on $X$ converges with respect to $\mathscr{I}$ to the $\mathscr{S}$-measurable function $f$ defined on $X$ if and only if every subsequence $\left\{f_{m_{n}}\right\}_{n \in N}$ of $\left\{f_{n}\right\}_{n \in N}$ contains a subsequence $\left\{f_{m_{p_{n}}}\right\}_{n \in N}$ converging to $f \mathscr{I}$-a.e. on $X$. We shall use the denotation $\underset{n}{\stackrel{\mathscr{S}}{\rightarrow}} f$ or $=\lim f_{n}$ with respect to $\mathscr{I}$.

We shall say that two $\mathscr{S}$-measurable functions $f$ and $g$ are equivalent if and only if $f-g$ is a null function (that is, if $f-g$ vanishes $\mathscr{I}$-a.e. on $X$ ).

It is not difficult to observe that the limit with respect to $\mathscr{I}$ is determined up to equivalent functions. In the above definition we can suppose also that all functions $f_{n}$ and $f$ are defined only $\mathscr{I}$-a.e. on $X$.

Observe also that if $(X, \mathscr{I}, \mu)$ is a finite measure space and $\mathscr{I} \subset \mathscr{S}$ is a $\sigma$-ideal of sets of measure zero, then convergence with respect to $\mathscr{I}$ is simply convergence in measure.

It is not difficult to verify that the following conditions are fulfilled: 
(L1) If $f_{n}=f$ for every $n$, then $f_{n} \underset{n \rightarrow \infty}{\stackrel{*}{\rightarrow}} f$.

(L2) If $f_{n} \underset{n \rightarrow \infty}{\stackrel{s}{\longrightarrow}} f$, then for every subsequence $\left\{f_{m_{n}}\right\}_{n \in N}$ of $\left\{f_{n}\right\}_{n \in N}$ we have $f_{m_{n}} \underset{n \rightarrow \infty}{\stackrel{s}{\longrightarrow}} f$.

(L3) If the sequence $\left\{f_{n}\right\}_{n \in N}$ is not convergent to $f$ with respect to $\mathscr{I}$, then there exists a subsequence $\left\{f_{m_{n}}\right\}_{n \in N}$, no subsequence of which converges to $f$ with respect to $\mathscr{I}$.

So the set of $\mathscr{S}$-measurable functions defined on $X$ equipped with convergence with respect to $\mathscr{I}$ is an $\mathscr{L} *$ space ([1]). One can define in an $\mathscr{L} *$ space the closure operation assuming that $f$ belongs to the closure of the set $A(f \in \bar{A})$ if and only if there exists a sequence $\left\{f_{n}\right\}_{n \in N}$ in $A$ such that $f_{n} \underset{n \rightarrow \infty}{\rightarrow} f$. The operation so defined has the following properties: $\bar{\varnothing}=\varnothing, A \subset \bar{A}, \overline{A \cup B}=\bar{A} \cup \bar{B}$ for every $A, B$, but the condition $\bar{A}=\bar{A}$ need not be fulfilled. This last condition is fulfilled if and only if the convergence has the following property:

(L4) If $f_{j} \underset{j \rightarrow \infty}{\stackrel{s}{\longrightarrow}} f$ and $f_{j, n} \underset{n \rightarrow \infty}{\stackrel{s}{\longrightarrow}} f_{j}$ for $j \in N$, then there exist two sequences of natural numbers $\left\{j_{p}\right\}_{p \in N},\left\{n_{p}\right\}_{p \in N}$ such that $f_{j, n_{p}} \underset{p \rightarrow \infty}{\stackrel{s}{\rightarrow}} f$.

If the space $\mathscr{L}^{*}$ fulfils the condition (L4), then we can equip the set of all $\mathscr{S}$-measurable functions with the topology determined by the closure operation described above. This topology is often called a Fréchet topology.

Observe that in the case of convergence with respect to $\mathscr{I}$ the condition (L4) is equivalent to the following condition:

(L4) If $f_{j} \underset{j \rightarrow \infty}{\stackrel{s}{\rightarrow}} f$ and $f_{j, n} \underset{n \rightarrow \infty}{\stackrel{s}{\rightarrow}} f_{j}$ for $j \in N$ and, moreover, $\left\{x: f_{j, n}(x) \neq f(x)\right\} \notin \mathscr{S}$, $\left\{x: f_{j}(x) \neq f(x)\right\} \notin \mathscr{I}$ for $j, n \in N$, then there exist an increasing sequence $\left\{j_{p}\right\}_{p \in N}$ of natural numbers and a sequence $\left\{n_{p}\right\}_{p \in N}$ of natural numbers such that $f_{j_{p}, n_{p}} \underset{p \rightarrow \infty}{\stackrel{s}{\rightarrow}} f$.

Definition 2. We shall say that a pair $(\mathscr{S}, \mathscr{A})$ fulfils the condition $(\mathrm{E})$ if and only if for every set $D \in \mathscr{S}_{-\mathscr{I}}$ and for every double sequence $\left\{B_{j, n}\right\}_{j, n \in \mathbb{N}}$ of $\mathscr{S}_{\text {-measur- }}$ able sets such that

$$
\begin{aligned}
& \text { (a) } B_{j, n} \subset B_{j, n+1} \text { for } j, n \in N, \\
& \text { (b) } \bigcup_{n=1}^{\infty} B_{j, n}=D \text { for } j \in N
\end{aligned}
$$

there exist an increasing sequence $\left\{j_{p}\right\}_{p \in N}$ of natural numbers and a sequence $\left\{n_{p}\right\}_{p \in N}$ of natural numbers such that $\bigcap_{p=1}^{\infty} B_{j_{p}, n_{p}} \notin \mathscr{\Phi}$.

THEOREM 1. Suppose that every family of disjoint sets in $\mathscr{S}-\mathscr{S}$ is at most denumerable. Then the set of all $\mathscr{S}$-measurable real functions is equipped with the Fréchet topology generated by the convergence with respect to $\mathscr{I}$ if and only if the pair $(\mathscr{S}, \mathscr{A})$ fulfils the condition (E).
Proof. Sufficiency. We can suppose, choosing the subsequences if necessary, that every sequence $\left\{f_{j, n}\right\}_{n \in N}$ is convergent $\mathscr{F}$-a.e. to $f_{j}$, and the sequence $\left\{f_{j}\right\}_{j \in N}$ converges $\mathscr{I}$-a.e. to $f$. Hence there exist a set $A \in \mathscr{I}$ and sets $A_{j} \in \mathscr{I}$ for $j \in N$ such that $f_{j, n}\left|X-A_{j} \underset{n \rightarrow \infty}{\rightarrow} f_{j}\right| X-A_{j}$ for every $j \in N$ and $f_{j}|X-A \underset{j \rightarrow \infty}{\rightarrow} f| X-A$. Put $D=X-\left(\bigcup_{j=1}^{\infty} A_{j} \cup A\right)$. Then $D \in \mathscr{S}-\mathscr{I}$. Let

$$
B_{j, n}=\left\{x \in D:\left|f_{j, k}(x)-f_{j}(x)\right|<1 / j \text { for every } k \geqslant n\right\} \text { for } j, n \in N .
$$

We have $B_{j, n} \subset B_{j, n+1}$ for $j, n \in N$ and $\bigcup_{n=1}^{\infty} B_{j, n}=D$ for every $j$. From the condition (E) it follows that there exist an increasing sequence $\left\{j_{p}\right\}_{p \in N}$ of natural numbers and a sequence $\left\{n_{p}\right\}_{p \in N}$ of natural numbers such that $\bigcap_{p=1}^{\infty} B_{j_{p}, n_{p}} \notin \mathscr{F}$. Let $x \in \bigcap_{p=1}^{\infty} B_{j_{p}, n_{p}}$. Then

$$
\left|f_{j_{p}, n_{p}}(x)-f_{j_{p}}(x)\right|<1 / j_{p} \quad \text { for every } p .
$$

Simultaneously $f_{j_{p}}(x) \underset{p \rightarrow \infty}{\rightarrow} f(x)$, because $x \in D$. Hence $f_{j_{p}, n_{p}}(x) \underset{p \rightarrow \infty}{\rightarrow} f(x)$. Then there exists. a sequence $\left\{f_{j, n_{p}}\right\}_{p \in N}$ such that

$$
f_{j_{p}, n_{p}}(x) \underset{p \rightarrow \infty}{\rightarrow} f(x) \text { for } \quad x \in \bigcap_{p=1}^{\infty} B_{j_{p}, n_{p}}=B^{(1)} \phi \mathscr{I} .
$$

Put $f_{1,1}^{(1)}=f_{J_{1}, n_{1}}, f_{1,2}^{(1)}=f_{f_{1, n_{1}+1}}, \ldots, f_{1}^{(1)}=f_{f_{1}} ; f_{2,1}^{(1)}=f_{J_{2}, n_{2}}, f_{2,2}^{(1)}=f_{J_{2}, n_{2}+1}, \ldots, f_{2}^{(1)}$ $=f_{j_{2}} ; \ldots ; f_{k, 1}^{(1)}=f_{j_{k}, n_{k}}, f_{k, 2}^{(1)}=f_{j_{k}, n_{k}+1}, \ldots, f_{k}^{(1)}=f_{j_{k}} ; \ldots$ It is easy to see that for every sequence $\left\{m_{k}\right\}_{k \in N}$ of natural numbers $f_{k, m_{k}}^{(1)}(x) \underset{k \rightarrow \infty}{\rightarrow} f(x)$ for $x \in B^{(1)}$. Indeed, one can prove convergence as before, observing only that $f_{k, m_{k}}^{(1)}=f_{j_{k}, n_{k}+m_{k}-1}$ and using the definition of $B_{j, n}$.

We proceed further by transfinite induction. Suppose that for every ordinal numbers $\alpha<\eta$, where $\eta$ is a countable ordinal, we have defined a double sequence $\left\{f_{j, n}^{(\alpha)}\right\}_{j, n \in N}$ of $\mathscr{S}$-measurable functions and a set $B^{(\alpha)} \in \mathscr{S}$ such that the sequence $\left\{B^{(\alpha)}\right\}_{\alpha<\eta}$ is nondecreasing and for every sequence $\left\{m_{k}\right\}_{k \in N}$ of natural numbers ${ }_{k, m_{k}}^{(\alpha)}(x) \underset{k \rightarrow \infty}{\rightarrow} f(x)$ for $x \in B^{(\alpha)}$ and if $\alpha_{1}<\alpha_{2}<\eta$, then every sequence $\left\{f_{\left.k, m_{k}\right\}_{k \in N}}^{\left(\alpha_{2}\right\}}\right.$ is almost a subsequence of some sequence $\left\{f_{k, l_{k}}^{\left(\alpha_{1}\right)}\right\}_{k \in N}$ (that is $\left\{f_{k, m_{k}}^{\left(\alpha_{2}\right)}\right\}_{k \in N}$ has at most a finite number of terms not belonging to $\left\{f_{\left.k, l_{k}\right)}^{(\alpha, 1)}\right\}_{k \in N}$. If $\eta$ has a predecessor and $D_{\eta-1}$ $=D-B^{(\eta-1)} \in \mathscr{I}$, then every sequence of the form $\left\{f_{k_{m}}^{(\eta-1)}\right\}_{k \in N}$ is convergent $\mathscr{I}$-a.e. on $X$ (and so also with respect to $\mathscr{A}$ ) to the function $f$ and the condition (L4') is fulfilled. Suppose now that $D_{\eta-1} \notin \mathscr{J}$. Put

$$
B_{j, n}^{(n-1)}=\left\{x \in D_{n-1}:\left|f_{j, k}^{(n-1)}(x)-f_{j}^{(n-1)}(x)\right|<1 / j \text { for every } k \geqslant n\right\} .
$$

We have

and

$$
B_{j, n}^{(\eta-1)} \subset B_{j, n+1}^{(\eta-1)} \text { for } j, n \in N
$$

$\bigcup_{n=1}^{\infty} B_{j, n}^{(n-1)}=D_{n-1} \quad$ for every $j$. 
From the condition (E) it follows that there exist an increasing sequence $\left\{j_{p}\right\}_{p \in N}$ of natural numbers and a sequence $\left\{n_{p}\right\}_{p \in N}$ of natural numbers such that $\bigcap_{p=1}^{\infty} B_{j_{p}, n_{p}}^{(\eta-1)} \notin \mathcal{I}$. As before, one can prove that $f_{j_{p}, n_{p}}^{(\eta-1)}(x) \underset{p \rightarrow \infty}{\rightarrow} f(x)$ for $x \in \bigcap_{p=1}^{\infty} B_{j_{p}, n_{p}}^{(\eta-1)}$. Put

$$
B^{(\eta)}=B^{(\eta-1)} \cup \bigcap_{p=1}^{\infty} B_{j_{p}, n_{p}}^{(\eta-1)}
$$

Then $f_{j_{p}, n_{p}}^{(\eta-1)}(x) \underset{p \rightarrow \infty}{\rightarrow} f(x)$ for $x \in B^{(\eta)}$ and $B^{(\eta)}-B^{(\eta-1)} \notin \mathscr{S}$.

If $\eta$ does not have a predecessor but there exists an ordinal number $\alpha<\eta$ such that $D_{\alpha}=D-B^{(\alpha)} \in \mathscr{I}$, then every sequence of the form $\left\{f_{k, m_{k}}^{(\alpha)}\right)_{k \in N}$ is convergent $\mathscr{I}$-a.e. on $X$ (and so also with respect to $\mathscr{I}$ ) to the function $f$ and the condition (L4') is fulfilled. Suppose now that $D_{\alpha} \notin \mathscr{I}$ for $\alpha<\eta$ and let $\left\{\alpha_{p}\right\}_{p \in N}$ be an increasing sequence of ordinal numbers converging to $\eta$. Put $B^{(\eta)}=\bigcup_{\alpha<\eta} B^{(\alpha)}$. Obviously $B^{(\eta)}=\bigcup_{p=1}^{\infty} B^{\left(\alpha_{p}\right)}$. We shall define a double sequence of functions in the following way: $f_{1,1}^{(\eta)}=f_{1,1}^{\left(\gamma_{1}\right)}, f_{1,2}^{(\eta)}=f_{1,2}^{\left(\alpha_{1}\right)}, \ldots, f_{1}^{(\eta)}=f_{1}^{\left(\alpha_{1}\right)} ; f_{2,1}^{(\eta)}=f_{2,2}^{\left(\alpha_{2}\right)}, f_{2,2}^{(\eta)}=f_{2,3}^{\left(\alpha_{2}\right)}, \ldots, f_{2}^{(\eta)}=f_{2}^{\left(\alpha_{2}\right)} ; \ldots ;$ $f_{k, 1}^{(\eta)}=f_{k, k}^{\left(\alpha_{k}\right)}, f_{k, 2}^{(\eta)}=f_{k, k+1}^{\left(\alpha_{k}\right)}, \ldots, f_{k}^{(\eta)}=f_{k}^{\left(\alpha_{k}\right)} ; \ldots$ Then for every sequence $\left\{m_{k}\right\}_{k \in N}$ of natural numbers we have $f_{k, m_{k}}^{(\eta)}(x) \underset{k \rightarrow \infty}{\rightarrow} f(x)$ for $x \in B^{\left(\eta_{p}\right)}$. Indeed, if $x \in B^{(\eta)}$, then there exists a number $p$ such that $x \in B^{\left(\alpha_{p}\right)}$. From the definition of the sequence $\left\{f_{j, n}^{(\eta)}\right\}_{j, n \in N}$ it follows that there exists a number $K(p)$ such that for every $k>K(p)$ the function $f_{k, m_{k}}^{(\eta)}$ is an element of some sequence $\left\{f_{l_{k}, p_{k}}^{\left(\alpha_{p}\right)}\right\}_{k \in N}$ converging to $f$ on the set $B^{\left(\alpha_{p}\right)}$.

We shall show that there exists an ordinal number $\beta<\Omega$ (where $\Omega$ stands for the smallest nondenumerable ordinal) such that $D-B^{(\beta)} \in \mathscr{I}$, and so our procedure finishes at some step. Suppose that $D-B^{(\alpha)} \notin \mathscr{I}$ for every $\alpha<\Omega$. From the definition of $B^{(\alpha)}$ it follows that if $\eta<\Omega$ has a predecessor, then $B^{(\eta)}-B^{(\eta-1)} \notin \mathscr{I}$. Put $C_{1}=B^{(1)}, C_{\alpha}=B^{(\alpha)}-B^{(\alpha-1)}$ for an ordinal having a predecessor. Then $C_{\alpha} \notin \mathscr{I}$ and $C_{\alpha} \cap C_{\eta}=\varnothing$ if $\alpha, \eta<\Omega, \alpha \neq \eta$ and $\alpha, \eta$ have predecessors. The set of all countable ordinals having predecessors is nondenumerable, which contradicts the assumption.

The sufficiency of the condition (E) is proved.

Necessity. Suppose that (E) is not fulfilled. So there exist a set $D \in \mathscr{S}-\mathscr{F}$ and a double sequence $\left\{B_{j, n}\right\}_{j, n \in N}$ fulfilling the conditions (a) and (b) and such that for every increasing sequence $\left\{j_{p}\right\}_{p \in N}$ of natural numbers and for every sequence $\left\{n_{p}\right\}_{p \in N}$ of natural numbers we have $\bigcap_{p=1}^{\infty} B_{j_{p}, n_{p}} \in \mathscr{F}$. Put $f_{j, n}(x)=\chi_{D-B_{j, n}}(x)+1 / j$ for $j, n \in N$. Then it is easy to see that $\lim _{n \rightarrow \infty} f_{j, n}^{p=1}=f_{j}=1 / j$ for every $j$ and $\lim _{j \rightarrow \infty} f_{j}=f=0$

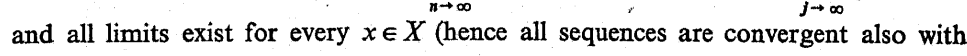
respect to $\mathscr{I}$.

Let $\left\{j_{p}\right\}_{p \in N}$ be an arbitrary increasing sequence of natural numbers and let $\left\{n_{p}\right\}_{p \in N}$ be an arbitrary sequence of natural numbers. For $x \in X$ the sequence $\left\{f_{j, n_{p}}(x)\right\}_{p \in N}$ does not converge to zero if and only if

$$
x \in \bigcap_{k=1}^{\infty} \bigcup_{p>k}\left(D-B_{j_{p}, n_{p}}\right)=D-\bigcup_{k=1}^{\infty} \bigcap_{p>k} B_{j_{p}, n_{p}} .
$$

The last set does not belong to the $\sigma$-ideal $\mathscr{I}$, and so the sequence $\left\{f_{j, n_{p}}\right\}_{p \in N}$ does not converge to zero $\mathscr{I}$-a.e. on $X$. From the arbitrariness of this sequence it follows that the condition (L4') is not fulfilled. This ends the proof.

Let us mention that the condition concerning denumerability of every disjoint family in $\mathscr{S}-\mathscr{I}$ is sometimes called (in the language of Boolean algebras) the countable chain condition.

EXAMPLE 1. If $(X, \mathscr{P},|\cdot|)$ is a finite measure space and $\mathscr{I}$ is a $\sigma$-ideal of all sets of measure zero, then it is well known that convergence with respect to $\mathscr{I}$ (convergence in measure) yields the topology. Hence the pair $(\mathscr{S}, \mathscr{A})$ fulfils the condition (E). It is well known that the countable chain condition is fulfilled. Also it is possible to prove the condition (E) directly. Indeed, let $D \in \mathscr{S}-\mathscr{I}$ and let $\left\{B_{j, n}\right\}_{j, n \in N}$ be a double sequence of measurable sets fulfilling (a) and (b). Then we have $|D|>0$ and it suffices to put $j_{p}=p$ for every natural $p$ and to choose $n_{p}$ in such a way that $\left|D-B_{p, n_{p}}\right|<|D| / 3^{p}$. Hence $\prod_{p=1}^{\infty} B_{p, n_{p}}|>| D \mid / 2>0$.

EXAMPLE 2. If $(X, \mathscr{L})$ is a measurable space and $\mathscr{I} \subset \mathscr{S}$ is a maximal $\sigma$-ideal, then the countable chain' condition is fulfilled (every disjoint family in $\mathscr{S}-\mathscr{I}$ can have at most one element). We shall prove that the condition (E) also holds. Let $D \in \mathscr{S}-\mathscr{I}$ and let $\left\{\boldsymbol{B}_{j, n}\right\}_{j, n \in N}$ be a double sequence of $\mathscr{S}$-measurable sets fulfilling (a) and (b). Put $j_{p}=p$ for every natural $p$ and choose $n_{p}$ in such a way that $B_{p, n_{p}} \notin \mathscr{I}$ (it is possible for every $p$ ). Then $\bigcap_{p=1}^{\infty} B_{p, n_{p}} \phi \mathscr{F}$, because

$$
X-\bigcap_{p=1}^{\infty} B_{p, n_{p}}=\bigcup_{p=1}^{\infty}\left(X-B_{p, n_{p}}\right) \in \mathscr{I} .
$$

EXAMPLE 3. Let $X=[0,1]$, let $\mathscr{S}$ be a class of sets having the Baire property and let $\mathscr{I}$ be a class of sets of the first category. It is well known that the countable chain condition is fulfilled. Constructing a double sequence of functions, we shall show that the condition (E) does not hold. Let

$$
f_{j, n}(x)=\chi_{A_{\jmath, n}}(x)+1 / j, \text { where } A_{j, n}=[0,1] \cap \bigcup_{j=1}^{j-1}\left(i / j-a_{n}, i / j+a_{n}\right),
$$

$\left\{a_{n}\right\}_{n \in N}$ is a decreasing sequence of numbers tending to zero and $f_{j}(x)=1 / j$ for $j ; n \in N$. Then $\lim f_{j, n}(x)=1 / j$ if $x \neq i \mid j, i=1, \ldots, j-1$ for every $j$, so $f_{j, n} \underset{n \rightarrow \infty}{\underset{\prime}{\longrightarrow}} f_{j}$. Also $f_{j} \underset{J \rightarrow \infty}{\stackrel{\leftrightarrow}{\longrightarrow}} \stackrel{n \rightarrow \infty}{f} \equiv 0$. Simultaneously we have $\left\{x: f_{j, n}(x) \neq f(x)\right\} \notin \mathscr{I}$ and $\left\{x: f_{j}(x) \neq f(x)\right\} \notin \mathscr{I}$ 
for $j, n \in N$. Let $\left\{j_{p}\right\}_{p \in N}$ be an increasing sequence of natural numbers and let $\left\{n_{p}\right\}_{p \in N}$ be an arbitrary sequence of natural numbers. We shall show that

$$
\left\{x: \sim\left(\lim _{\boldsymbol{p} \rightarrow \infty} f_{j_{\mathbf{p}, n_{p}}}(x)=0\right)\right\} \notin \mathscr{I} .
$$

From the arbitrariness of $\left\{j_{p}\right\}_{p \in N}$ and $\left\{n_{p}\right)_{p \in N}$ we conclude that no sequence $\left\{f_{j_{p}, n_{p}}\right\}_{p \in N}$ converges to $f$ with respect to $\mathscr{I}$. Observe that

$$
\begin{aligned}
\left\{x: \sim\left(\lim _{p \rightarrow \infty} f_{j_{p}, n_{p}}(x)=0\right)\right\} & \supset\left\{x: \underset{p}{\limsup } f_{j_{p}, n_{p}}(x) \geqslant 1\right\} \\
& \supset \bigcap_{M=1}^{\infty} \bigcup_{p=M+1}^{\infty}\left\{x: f_{j_{p}, n_{p}}(x) \geqslant 1\right\} \\
& =\bigcap_{M=1}^{\infty} \bigcup_{p=M+1}^{\infty} A_{j_{p}, n_{p}} .
\end{aligned}
$$

For every $M$ the set $\bigcup_{p=M+1}^{\infty} A_{j_{p}, n_{p}}$ is open and dense in $[0,1]$, and so

$$
\left\{x: \sim\left(\lim _{p \rightarrow \infty} f_{j_{p}, h_{\dot{p}}}(x)=0\right)\right\}
$$

is a residual set and does not belong to $\mathscr{I}$.

EXAMPLE 4. Let $X$ be an arbitrary nondenumerable set, $\mathscr{S}=2^{X}$ and $\mathscr{I}=\{\varnothing\}$. Then convergence with respect to $\mathscr{I}$ is simply convergence at every point. It is well known that in this case this kind of convergence does not yield a topology. However the condition (E) is fulfilled. Indeed, let $D \in \mathscr{S}-\mathscr{I}$ and let $\left\{B_{j, n}\right\}_{j, n \in N}$ be a double sequence of sets fulfilling (a) and (b). $D$ is nonempty; let $x_{0} \in D$. It suffices to put $j_{p}=p$ and to choose $n_{p}$ in such a way that $x_{p} \in B_{p, n_{p}}$. Hence $x_{0} \in \bigcap_{p=1}^{\infty} B_{p, n_{p}}$, so $\bigcap_{p=1}^{\infty} B_{p, n_{p}} \notin \mathscr{I}$.

Observe that the pair $(\mathscr{S}, \mathscr{S})$ does not fulfil the countable chain condition. So this condition in Theorem 1 is important.

\section{II}

If $X$ is an arbitrary non-empty set, $\mathscr{S}$ - a fixed $\sigma$-field of subsets of $X$ and $\mathscr{I} \subset \mathscr{S}$ - a $\sigma$-ideal, then identifying the sets $A, B \in \mathscr{S}$ if and only if $A \triangle B \in \mathscr{I}$ we obtain a quotient Boolean algebra $\mathscr{S} / \mathscr{F}$. The class including a set $A$ will be denoted by $[A]$. For classes $[A],[B] \in \mathscr{S} / \mathscr{I}$ the notation $[A] \subset[B]$ means that $A_{1}-B_{1} \in \mathscr{I}$ for every (or, equivalently, for some) $A_{1} \in[A]$ and $B_{1} \in[B]$. In virtue of Theorem 21.1 from [5], p. $74 \mathscr{S} / \mathscr{I}$ is a $\sigma$-complete Boolean algebra.

Denote by $[f]$ the class of all $\mathscr{S}$-measurable real functions equivalent to an $\mathscr{S}$-measurable function $f$. For classes $[f]$ and $[g]$ the notation $[f] \leqslant[g]$ means that $f_{1}(x) \leqslant g_{1}(x) \mathscr{I}$-a.e. on $X$ for every (or, equivalently, for some) $f_{1} \in[f]$ and $g_{1} \in[g]$.
LEMMA 1. The Boolean algebra $\mathscr{S} \mid \mathscr{S}$ is a complete lattice if and only if the family $\mathfrak{D}$ of all equivalence classes of real $\mathscr{S}$-measurable functions is a complete lattice.

The proof of the above lemma is essentialy the same as the proof of Lemma 1 in [3], p. 106, and so we shall omit it.

LEMMA 2. If a $\sigma$-complete Boolean algebra $\mathscr{S} \mid \mathscr{S}$ fulfils the countable chain condition, then $\mathscr{S} / \mathscr{I}$ is a complete lattice.

Proof. It follows immediately from Theorem 20.5 from [5], p. 72.

COROLLARY. If a $\sigma$-complete Boolean algebra $\mathscr{S} \mid \mathscr{I}$ fulfils the countable chain condition, then $\mathfrak{M}$ is a complete lattice.

In this part of the paper we shall denote both $[f]$ and its elements by $f$, hoping that no ambiguity will result and that the reader will make suitable distinctions according to the context. Also we shall suppose that $\mathscr{S} \mid \mathscr{I}$ fulfils the countable chain condition.

We shall now define an equivalence relation for sequences of $\mathscr{S}$-measurable functions. Let $\left\{f_{n}\right\}_{n \in N}$ be equivalent to $\left\{g_{n}\right\}_{n \in N}$ if and only if $\left\{f_{n}-g_{n}\right\}_{n \in N}$ converges with respect to $\mathscr{I}$ to zero. Let $\left\{f_{n}\right\}_{n \in N}$ be a sequence of $\mathscr{S}$-measurable functions and let $\mathscr{F}$ be the equivalence class including $\left\{f_{n}\right\}_{n \in N}$.

Definition 3. We shall say that $U \in \mathfrak{M}(L \in \mathfrak{M})$ is an upper (lower) limit of a sequence $\left\{f_{n}\right\}_{n \in N}$ with respect to $\mathscr{I}$ if and only if

$$
\begin{aligned}
& U=\inf \left[\limsup _{n} g_{n}:\left\{g_{n}\right\}_{n \in N} \in \mathscr{F}\right] \\
& \left(L=\sup \left[\liminf g_{n}:\left\{g_{n}\right\}_{n \in N} \in \mathscr{F}\right]\right) .
\end{aligned}
$$

The existence of $U$ and $L$ follows from the corollary and from the fact that for every sequence $\left\{g_{n}\right\}_{n \in N}$ of $\mathscr{S}$-measurable functions the function limsupg $g_{n}$ and $\liminf g_{n}$ is also $\mathscr{S}$-measurable.

LEMMA 3. If $U$ and $L$ are upper and lower limits with respect to $\mathscr{I}$ of a sequence $\left\{f_{n}\right\}_{n \in N}$, then $L \leqslant U$.

Proof. Suppose that $U(x)<L(x)$ on a set $B \notin \mathscr{I}$. Then there exists a sequence $\left\{h_{n}\right\}_{n \in N} \in \mathscr{F}$ such that $\limsup h_{n}(x)<L(x)$ on the set $C \notin \mathscr{I}$ and there exists a sequence $\left\{g_{n}\right\}_{n \in N} \in \mathscr{F}$ such that $\limsup _{n} h_{n}(x)<\liminf _{n} g_{n}(x)$ on the set $D \notin \mathscr{I}$. We have

$$
D=\bigcup_{\substack{w \in W \\ w>0}}\left\{x: \limsup _{n} h_{n}(x)+w<\liminf _{n} g_{n}(x)\right\},
$$

where $W$ is the set of all rational numbers, and so there exists a number $w_{0}>0$ such that the set $\left\{x: \limsup h_{n}(x)+w_{0}<\liminf g_{n}(x)\right\}$ does not belong to $\mathscr{I}$. Obviously

$$
\begin{aligned}
\left\{x: \limsup _{n} h_{n}(x)+w_{0}<\liminf _{n}(x)\right\} \\
\quad=\bigcup_{n=1}^{\infty}\left\{x: \sup \left(h_{n}(x), h_{n+1}(x), \ldots\right)+w_{0}<\inf \left(g_{n}(x), g_{n+1}(x), \ldots\right)\right\}
\end{aligned}
$$


hence there exists a natural number $n_{0}$ such that the set

$$
E=\left\{x: \sup \left(h_{n_{0}}(x), h_{n_{0}+1}(x), \ldots\right)+w_{0}<\inf \left(g_{n_{0}}(x), g_{n_{0}+1}(x), \ldots\right)\right\}
$$

does not belong to $\mathscr{I}$. Hence for every $x \in E$ and for every $n \geqslant n_{0}$ we have $g_{n}(x)-h_{n}(x)>w_{0}>0$, and so a sequence $\left\{g_{n}\right\}_{n \in N}$ is not equivalent to $\left\{h_{n}\right\}_{n \in N}-$ a contradiction.

LEMMA 4. Let $\left\{f_{n}\right\}_{n \in N}$ be a sequence of $\mathscr{S}$-measurable functions. If $\left\{f_{n}\right\}_{n \in N}$ does not converge to zero with respect to $\mathscr{F}$, then at least one of the following conditions is fulfilled:

1. there exist a subsequence $\left\{f_{m_{n}}\right\}_{n \in N}$ of $\left\{f_{n}\right\}_{n \in N}$, a set $A_{0} \in \mathscr{S}-\mathscr{I}$ and a natural number $k_{0}$ such that for every subsequence $\left\{f_{m_{p_{n}}}\right\}_{n \in N}$ of $\left\{f_{m_{n}}\right\}_{n \in N}$ we have $\limsup f_{m_{p_{n}}}(x) \geqslant 1 / k_{0}$-a.e. on $A_{0}$;

2. there exist a subsequence $\left\{f_{m_{n}}\right\}_{n \in N}$ of $\left\{f_{n}\right\}_{n \in N}$, a set $A_{0} \in \mathscr{S}-\mathscr{I}$ and a natural number $k_{0}$ such that for every subsequence $\left\{f_{m_{p_{n}}}\right\}_{n \in N}$ of $\left\{f_{m_{n}}\right\}_{n \in N}$ we have $\liminf f_{m_{p_{n}}}(x) \leqslant-1 / k_{0} \mathscr{T}$-a.e. on $A_{0}$.

"Proof. Suppose on the contrary that for every subsequence $\left\{f_{m_{n}}\right\}_{n \in N}$ of $\left\{f_{n}\right\}_{n \in N}$, for every set $A \in \mathscr{S}-\mathscr{I}$ and for every natural number $k$ there exists a subsequence $\left\{f_{m_{p_{n}}}\right\}_{n \in N}$ of $\left\{f_{m_{n}}\right\}_{n \in N}$ such that $\limsup f_{m_{p_{n}}}(x)<1 / k$ on the set $A^{\prime} \subset A$ such that $A^{\prime} \in \mathscr{S}-\mathscr{I}$ and that for every subsequence $\left\{f_{m_{n}}\right\}_{n \in N}$ of $\left\{f_{n}\right\}_{n \in N}$, for every set $A \in \mathscr{S}-\mathscr{I}$ and for every natural number $k$ there exists a subsequence $\left\{f_{m_{p_{n}}}\right\}_{n \in N}$ of $\left\{f_{m_{n}}\right\}_{n \in N}$ such that $\liminf f_{m_{p_{n}}}(x)>-1 / k$ on the set $A^{\prime \prime} \subset A$ such that $A^{\prime \prime} \in \mathscr{S}-\mathscr{I}$.

As a first step we shall prove that under this supposition for every subsequence $\left\{f_{m_{n}}\right\}_{n \in N}$ there exists a subsequence $\left\{\hat{f}_{n}^{(1)}\right\}_{n \in N}$ of $\left\{f_{m_{n}}\right\}_{n \in N}$ such that $\limsup _{n} \hat{f}_{n}^{(1)}(x)<1$ S-a.e. on $X$.

Let $\left\{f_{m_{n}}\right\}_{n \in N}$ be an arbitrary subsequence of $\left\{f_{n}\right\}_{n \in N}$. Put $A=X$ and $k=1$. There exists a subsequence $\left\{f_{n}^{(1)}\right\}_{n \in N}$ of $\left\{f_{m_{n}}\right\}_{n \in N}$ such that

$$
B_{1}=\left\{x: \underset{n}{\limsup } f_{n}^{(1)}(x)<1\right\} \notin \mathscr{I} \text {. }
$$

Observe that $B_{1} \in \mathscr{S}$.

Suppose that for every ordinal number $\alpha<\eta$, where $\eta<\Omega$, we have chosen a subsequence $\left\{f_{n}^{(\alpha)}\right\}_{n \in N}$ of $\left\{f_{m_{n}}\right\}_{n \in N}$ and a set $B_{\alpha} \in \mathscr{S}$ such that if $\alpha_{1}<\alpha_{2}<\eta$, then $\left\{f_{n}^{\left(\alpha_{2}\right)}\right\}_{n \in}$, is almost a subsequence of $\left\{f_{n}^{\left(\alpha_{1}\right)}\right\}_{n \in N}$, the sequence of sets $\left\{B_{\alpha}\right\}_{\alpha<\eta}$ is nondecreasing and $\lim \sup f_{n}^{(\alpha)}(x)<1$ for $x \in B_{\alpha}$. If $\eta$ has a predecessor and $X-B_{\eta-1} \in \mathscr{I}$, then we put $f_{n}^{(\eta)}=f_{n}^{(n-1)}$ for $n=1,2, \ldots$ If $X-B_{\eta-1} \notin \mathscr{I}$, then in virtue of the supposition we can choose a subsequence $\left\{f_{n}^{(n)}\right\}_{n \in N}$ of $\left\{f_{n}^{(n-1)}\right\}_{n \in N}$ such that $\lim \sup f_{n}^{(n)}(x)<1$ for $x \in B_{\eta}$ and $B_{\eta}-B_{\eta-1} \notin \mathscr{I}$. Indeed, it suffices to take $A=X-B_{\eta-1}{ }^{n}$ and $k=1$ If $\eta$ does not have a predecessor and there exists an ordinal number $\alpha<\eta$ such that $X-B_{\alpha} \in \mathscr{I}$, then we put $f_{n}^{(\eta)}=f_{n}^{(\alpha)}$ for $n=1,2, \ldots$ If $X-B_{\alpha} \notin \mathscr{I}$ for $\alpha<\eta$, then we choose an increasing sequence $\left\{\alpha_{k}\right\}_{k \in N}$ of ordinal numbers tending to $\eta$. Put $B_{\eta}=\bigcup_{\alpha<\eta} B_{\alpha}$. Then of course $B_{\eta}=\bigcup_{k=1}^{\infty} B_{\alpha_{k}}$. Let $\left\{f_{n}^{(\eta)}\right\}_{n \in N}$ be a diagonal subsequence chosen from the family $\left\{f_{n}^{\left(\alpha_{k}\right)}\right\}_{n \in N}, k=1,2, \ldots$ Then $\limsup f_{n}^{(\eta)}(x)<1$ for $x \in B_{\eta}$.

We shall show that there exists an ordinal number $\beta^{n}<\Omega$ such that $X-B_{\beta} \in \mathscr{I}$. Suppose that $X-B_{\alpha} \notin \mathscr{I}$ for every $\alpha<\Omega$. From the definition of sets $\left\{B_{\alpha}\right\}_{\alpha<\Omega}$ it follows that if $\eta<\Omega$ has a predecessor, then $B_{\eta}-B_{\eta-1} \phi \mathscr{I}$. Put $C_{1}=B_{1}, C_{\alpha}=B_{\alpha}-B_{\alpha-1}$, when $1<\alpha<\Omega$ is an ordinal number having a predecessor. Then $C_{\alpha} \phi \mathscr{I}$ and $C_{\alpha} \cap C_{\eta}=\varnothing$ if $\alpha \neq \eta, \alpha, \eta<\Omega, \alpha$ and $\eta$ are ordinal numbers having predecessors. We have thus obtained a contradiction with the countable chain condition. Hence there exists an ordinal number $\beta<\Omega$ such that $X-B_{\beta} \in \mathscr{I}$. If we put $\vec{f}_{n}^{(1)}=f_{n}^{(\beta)}$ for $n \in N$, we obtain a subsequence for which $\lim \sup \hat{f}_{n}^{(1)}(\lambda)<1 \mathscr{I}$-a.e. on $X$.

Repeating this argument for $k=2$ and for ${ }^{n}\left\{\hat{f}_{n}^{(1)}\right\}_{n \in N}$, we obtain a subsequence $\left\{\hat{f}_{n}^{(2)}\right\}_{n \in N}$ of $\left\{\hat{f}_{n}^{(1)}\right\}_{n \in N}$ such that lim sup $\hat{f}_{n}^{(2)}(x)<\frac{1}{2} \mathscr{I}$-a.e. on $X$. Proceeding similarly for $k=3,4, \ldots$ and then choosing a diagonal subsequence $\left\{f_{n}\right\}_{n \in N}$, one can prove without difficulty that $\limsup f_{n}(x) \leqslant 0 \mathscr{I}$-a.e. on $X$.

Using the second part of the supposition, we can choose a subsequence $\left\{f_{m_{p_{n}}}\right\}_{n \in N}$ of $\left\{f_{n}\right\}_{n \in N}$ such that liminf $f_{m_{p_{n}}}(x) \geqslant 0 \mathscr{I}$-a.e. on $X$.

Hence from an arbitrary subsequence $\left\{f_{m_{n}}\right\}_{n \in N}$ of $\left\{f_{n}\right\}_{n \in N}$ we can choose a subsequence $\left\{f_{m_{p_{n}}}\right\}_{n \in N}$ converging to zero $\mathscr{I}$-a.e. on $X$ - a contradiction.

THEOREM 2. Let $f$ and $f_{n}, n \in N$ be $\mathscr{S}$-measurable functions. A sequence $\left\{f_{n}\right\}_{n \in N}$ converges with respect to $\mathscr{I}$ to a function $f$ if and only if $U=L$, and then $f=U=L$.

Proof. Suppose that $\left\{f_{n}\right\}_{n \in N}$ converges to $f$ with respect to $\mathscr{I}$. Then a sequence $\left\{f_{n}\right\}_{n \in N}$ is equivalent to a sequence $\left\{g_{n}\right\}_{n \in N}$, where $g_{n}=f$ for $n=1,2, \ldots$ Hence $U \leqslant f$, because $\limsup g_{n}=f$. Similarly one can prove that $L \geqslant f$. So $f \leqslant L \leqslant U \leqslant f$ and $U=L$.

Suppose now that $U=L$. Without loss of generality we can suppose that $U \equiv 0$. Assume that $\left\{f_{n}\right\}_{n \in N}$ does not converge to zero with respect to $\mathscr{F}$ and that the first condition of Lemma 4 is fulfilled (in the remaining case the proof is similar).

Let $\left\{g_{n}\right\}_{n \in N}$ be a sequence equivalent to $\left\{f_{n}\right\}_{n \in N}$. Then $g_{n}=f_{n}+h_{n}, n \in N$, where $\left\{h_{n}\right\}_{n \in N}$ is a sequence of $\mathscr{S}$-measurable functions converging to zero with respect to $\mathscr{I}$. Let $\left\{f_{m_{n}}\right\}_{n \in N}$ be a subsequence, $A_{0}-$ a set and $k_{0}-$ a natural number described in Lemma 4 . There exists a subsequence $\left\{h_{m_{p_{n}}}\right\}_{n \in N}$ of $\left\{h_{m_{n}}\right\}_{n \in N}$ convergent to zero at every, $x \in X-C$, where $C \in \mathscr{I}$. Let $x \in A_{0}-C$. Then

$$
\limsup _{n}(x) \geqslant \limsup _{n} g_{m_{p_{n}}}(x)=\limsup \left(f_{m_{p_{n}}}(x)+h_{m_{p_{n}}}(x)\right) \geqslant 1 / k_{0}
$$

From the arbitrariness of $\left\{g_{n}\right\}_{n \in N}$ and from the definition of $U$ we have $U(x) \geqslant 1 / k_{0}$ I. -a.e. on $A_{0}$. This contradiction ends the proof.

2 - Fundamenta Mathematicae CXrr 


\section{III}

Let $X$ be an arbitrary non-empty set, $\mathscr{S}-$ a fixed $\sigma$-field of subsets of $X$ and $\mathscr{I} \subset \mathscr{S}$ - a $\sigma$-ideal.

Definition 4. We shall say that a pair $(\mathscr{S}, \mathscr{S})$ fulfils the condition $(\mathrm{F})$ if and only if for every set $D \in \mathscr{S}-\mathscr{I}$ and for every double sequence of $\mathscr{S}$-measurable sets $\left\{B_{j, n}\right\}_{j, n \in N}$ such that

(a) $B_{j, n} \subset B_{j, n+1}$ for $j, n \in N$,

(b) $\bigcup_{n=1}^{\infty} B_{j, n}=D$ for $j \in N$,

(c) $B_{i_{1}, J_{1}} \supset B_{i_{2}, j_{2}}$ if $i_{1}<i_{2}$ and $i_{1}+j_{1}=i_{2}+j_{2}$

there exists a sequence $\left\{n_{j}\right\}_{j \in N}$ of natural numbers such that $\limsup _{j} B_{j, n} \not \mathscr{I}$.

LEMMA 5. If a double sequence $\left\{S_{p, r}\right\}_{p, r \in N}$ of $\mathscr{S}$-measurable sets fulfils the conditions (a), (b) and (c) from Definition 4 , then there exists a double sequence $\left\{H_{m, n}\right\}_{m, n \in N}$ of $\mathscr{S}$-measurable sets such that $S_{p, r}=\bigcup_{m=p}^{p+r-1} \bigcup_{n=p}^{p+r-1} H_{m, n}$ for every $p, r \in N$.

Proof. Put $H_{n, n}=S_{n, 1}$ for $n \in N, H_{m, n}=S_{m, n-m+1}-\left(S_{m, n-m} \cup S_{m+1, n-m}\right)$ if $m<n, m, n \in N$ and $H_{m, n}=\varnothing$ if $m>n, m, n \in N$. We shall proceed by induction with respect to $r$. For $r=1$ the equality is obvious. Suppose that $S_{p, r}=\bigcup_{m=p}^{p+r-1} \bigcup_{n=p}^{p+r-1}$ $H_{m, n}$. Then

$$
\begin{aligned}
& \bigcup_{m=p}^{p+r} \bigcup_{n=p}^{p+r} H_{m, n} \\
&=\bigcup_{m=p}^{p+r-1} \bigcup_{n=p}^{p+r-1} H_{m, n} \cup H_{p, p+r} \cup H_{p+1, p+r} \cup \ldots \cup H_{p+r-1, p+r} \cup H_{p+r, p+r} \\
&= S_{p, r} \cup\left[S_{p, r+1}-\left(S_{p, r} \cup S_{p+1, r}\right)\right] \cup\left[S_{p+1, r}-\left(S_{p+1, r-1} \cup S_{p+2, r-1}\right)\right] \cup \ldots \\
& \ldots \cup\left[S_{p+r-1,2}-\left(S_{p+r-1,1} \cup S_{p+r, 1}\right)\right] \cup S_{p+r, 1} \\
&= S_{p, r+1},
\end{aligned}
$$

because from (c) it follows that $S_{p+r, r-1} \subset S_{p, r}, \ldots, S_{p+r-1,1} \subset S_{p, r}$ for $r>1, r \in N$ and for every $p \in N$.

THEOREM 3. Let $\left\{f_{m, n}\right\}_{m, n \in N}$ be a double sequence of $\mathscr{S}$-measurable real functions defined on $X$. The convergence $\mathscr{I}$-a.e. to f of all sequences $\left\{f_{m_{k}, n_{k}}\right\}_{k \in N}$ where $m_{k} \underset{k \rightarrow \infty}{\rightarrow} \infty$, $n_{k} \underset{k \rightarrow \infty}{\rightarrow} \infty$ implies the convergence $\mathscr{I}$-a.e. to $f$ of a double sequence $\left\{f_{m, n}\right\}_{m, n \in N}$ if and only if the pair $(\mathscr{S}, \mathscr{F})$ fulfils the condition (F).

Proof. Sufficiency. Assume that $f(x) \equiv 0$. Suppose that

$$
E=\left\{x: \sim\left(\lim _{m, n \rightarrow \infty} f_{m, m}(x)=0\right)\right\} \notin \mathscr{I} .
$$

For every $x \in E$ there exist a number $a_{x}>0$ and two sequences $\left\{m_{k}\right\}_{k \in N},\left\{n_{k}\right\}_{k \in N}$ of natural numbers tending to infinity such that $\left|f_{m_{k}, m_{k}}(x)\right| \geqslant a_{x}$ for every $k \in N$. Denote by $E_{a}$ the set of $x \in E$ for which there exist two sequences $\left\{m_{k}\right\}_{k \in N}$ and $\left\{n_{k}\right\}_{k \in N}$ of natural numbers tending to infinity such that $\left|f_{m_{k}, n_{k}}(x)\right| \geqslant a$ for every $k \in N$. We have $E=\bigcup_{i=1}^{\infty} E_{1 / i}$. There exists a natural number $i_{0}$ such that $E_{1 / i_{0}} \notin \mathscr{I}$. Denote $E_{1 / i_{0}}$ by $H$. Put $H_{m, n}=\left\{x:\left|f_{m, n}(x)\right| \geqslant 1 / i_{0}\right\} \cap H$ for $m, n \in N$ and $S_{p}=\bigcup_{m=p}^{\infty} \bigcup_{n=p}^{\infty} H_{m, n}$ for $p \in N$. Then it is easy to verify that $H=S_{p}$ for every $p \in N$. If we put $S_{p, r}=\bigcup_{m=p}^{p+r-1} \bigcup_{n=p}^{p+r-1} H_{m, n}$ then it is not difficult to prove that the double sequence $\left\{S_{p, r}\right\}_{p, r \in N}$ fulfils the conditions (a), (b) and (c) of Definition 4 with $D=H$. From the condition (F) it follows that there exists a sequence $\left\{r_{p}\right\}_{p \in N}$ such that $Q=\limsup S_{p, r_{p}} \notin \mathscr{I}$. Let $\left(m_{k}, n_{k}\right)$ be the $k$ th element of the following sequence:

$$
\begin{aligned}
&(1,1), \ldots,\left(1,1+r_{1}-1\right),(2,1), \ldots,\left(2,2+r_{1}-1\right), \ldots \\
& \ldots,\left(1+r_{1}-1,1\right), \ldots,\left(1+r_{1}-1,1+r_{1}-1\right), \\
&(2,2), \ldots,\left(2,2+r_{2}-1\right),(3,2), \ldots,\left(3,2+r_{2}-1\right) \ldots \\
& \ldots,\left(2+r_{2}-1,2\right), \ldots,\left(2+r_{2}-1,2+r_{2}-1\right), \\
&(p, p), \ldots,\left(p, p+r_{p}-1\right),(p+1, p), \ldots,\left(p+1, p+r_{p}-1\right), \ldots \\
& \ldots,\left(p+r_{p}-1, p\right), \ldots,\left(p+r_{p}-1, p+r_{p}-1\right),
\end{aligned}
$$

Then $m_{k} \rightarrow \infty$ and $n_{k} \rightarrow \infty$. If $x \in Q$, then $x$ belongs to infinitely many sets $S_{p, r_{p}}$, and so in the sequence $\left\{f_{m_{k}, n_{k}}(x)\right\}_{k \in N}$ there are infinitely many elements greater than $1 / i_{0}$; so the sequence $\left\{f_{m_{k}, n_{k}}\right\}_{k \in N}$ does not tend to zero $\mathscr{F}$-a.e. on $X$. This is a contradiction.

Necessity. Suppose that the condition $(F)$ is not fulfilled, and so there exist a set $D \in \mathscr{S}-\mathscr{I}$ and a double sequence $\left\{S_{p, r}\right\}_{p, r \in N}$ of $\mathscr{S}_{\text {-measurable sets fulfilling }}$ the conditions (a), (b) and (c) of Definition 4 and such that for every sequence $\left\{r_{p}\right\}_{p \in N}$ we have $\limsup S_{p, r} \in \mathscr{I}$. Let $\left\{H_{m, n}\right\}_{m, n \in N}$ be a double sequence of sets described in Lemma 5. Put $f_{m, n}(x)=\chi_{H_{m, n}}(x)$ for $m, n \in N$. We shall prove that for all sequences $\left\{m_{k}\right\}_{k \in N}$ and $\left\{n_{k}\right\}_{k \in N}$ of natural numbers tending to infinity we have $\lim f_{m_{k}, m_{k}}(x)=0$. $f$-a.e. on $X$. Indeed, the last assertion does not hold if and only if $x \in \limsup H_{m_{k}, n_{k}}$. Let $M^{*}=\left\{m_{k}: k \in N\right\}$ and $N^{*}=\left\{n_{k}: k \in N\right\}$. If $p \notin M^{*} \cup N^{*}$, then we put $r_{p}=1$. If $p \in M^{*} \cup N^{*}$ then we put

$$
r_{p}=\max \left(\max \left\{n_{k}: m_{k}=p\right\}, \max \left\{m_{k}: n_{k}=p\right\}\right)-p+1
$$

(we take zero as the max of the empty set). Observe that the sets $\left\{n_{k}: m_{k}=p\right\}$ and $\left\{m_{k}: n_{k}=p\right\}$ are both finite, because $m_{k} \underset{k \rightarrow \infty}{\rightarrow} \infty$ and $n_{k} \underset{k \rightarrow \infty}{\rightarrow} \infty$, and so the definition 
of $r_{p}$ is correct. From this definition it follows without difficulty that for every natural $k$ there exists a natural number $p$ such that $H_{m_{k}, n_{k}} \subset S_{p, r_{p}}$. Hence $\limsup H_{m_{k}, n_{k}}$ $\subset \lim \sup S_{p, r_{p}} \in \mathscr{I}$, and the convergence $\mathscr{I}$-a.e. to zero of the sequence $\left\{f_{m_{k}, n_{k}}\right\}_{k \in N}$ is proved.

Simultaneously it is easy to see that the sequence $\left\{f_{m, n}\right\}_{m, n \in N}$ does not tend to zero for any $x \in D$.

W. Sierpiński in [4] has proved that if $X=[0,1], \mathscr{S}$ is a $\sigma$-field of sets measurable in the sense of Lebesgue and $\mathscr{I}$ is a $\sigma$-ideal of sets of Lebesgue measure zero, then the convergence a.e. of every sequence $\left\{f_{m_{k}, n_{k}}\right\}_{k \in N}$ for $\left\{m_{k}\right\}_{k \in N}$ and $\left\{n_{k}\right\}_{k \in N}$ tending to infinity implies the convergence a.e. of a double sequence $\left\{f_{m, n}\right\}_{m, n \in N}$. So in this case the pair $(\mathscr{S}, \mathscr{F})$ fulfils the condition $(F)$. The proof in [4] is valid also in the case of an arbitrary $\sigma_{m}$-finite measure space $(X, \mathscr{S}, \mu)$ and the $\sigma$-ideal of sets of measure $\mu$ zero.

Observe that if the pair $(\mathscr{S}, \mathscr{I})$ fulfils the condition (E), then it fulfils also the condition $(\mathrm{F})$. Now we shall show an example of a pair $(\mathscr{S}, \mathscr{T})$ fulfilling $(\mathrm{F})$ different from the pair ( $\sigma$-field of all measurable sets in $\sigma$-finite measure space, $\sigma$-ideal of null sets).

Let $X$ be a complete ànd separable metric space, $\mathscr{S}-a \sigma$-field of sets having the Baire property and $\mathscr{I}-a \sigma$-ideal of sets of the first category.

Theorem 4. A pair $(\mathscr{S} ; \mathscr{A})$ fulfils the condition $(\mathrm{F})$.

Proof. Let $D \in \mathscr{S}-\mathscr{I}$ and let $\left\{B_{j, n}\right\}_{j, n \in N}$ be a double sequence of sets belonging to $\mathscr{S}$ and fulfilling the conditions (a), (b) and (c) of Definition 4. We have $D=G \triangle P$, where $G$ is an open and non-empty set and $P$ is a set of the first category. Let $K(x, r)$ be a ball with the centre $x$ and the radius $r>0$ included in $G$. It suffices to show that there exists a sequence $\left\{n_{j}\right\}_{j \in N}$ of natural numbers such that $\limsup \left(B_{j, n_{j}} \cap K(x, r)\right.$ is residual in $K(x, r)$.

Put $C_{j, n}=B_{\jmath, n} \cap K(x, r)$ for $j, n \in N$. Then $C_{j, n} \in \mathscr{S}$ for every $j, n \in N$, and so we have $C_{J, n}=G_{j, n} \Delta P_{j, n}$, where $G_{j, n}$ is open and $P_{j, n}$ is of the first category for every $j, n \in N$. We have

and

$$
\underset{j}{\limsup } C_{j, n_{j}}=\bigcap_{k=1}^{\infty} \bigcup_{j=k}^{\infty} C_{j, n,}
$$

$$
\bigcup_{j=k}^{\infty} C_{j, n j} \supset \bigcup_{j=k}^{\infty} G_{j, n j}-\bigcup_{j=k}^{\infty} P_{j, n j},
$$

and so it suffices to choose a sequence $\left\{n_{j}\right\}_{j \in N}$ in such a way that for every $k \in N$ the set $\bigcup_{j=k}^{\infty} G_{j, n_{j}}$ is dense in $K(x, r)$.

From the condition (b) it follows that $\bigcup_{n=1}^{\infty} C_{j, n}=D \cap K(x, r)$, and so it is not difficult to see that $\bigcup_{n=1}^{\infty} G_{j, n}$ is dense in $K(x, r)$ for every $j \in N$. Let $\left\{G_{j}: j \in N\right\}$ be a basis for a subspace topology in $K(x, r)$ such that $\left\{G_{j}: j=k, k+1, \ldots\right\}$ is also a basis for every $k \in N$ (the existence of such a basis easily follows from the separability). For every $j \in N$ we have $G_{j} \cap \bigcup_{n=1}^{\infty} G_{j ; n} \neq \varnothing$, because the set $\bigcup_{n=1}^{\infty} G_{j, n}$ is dense in $K(x, r)$. So for every $j \in N$ there exists a natural number $n_{j}$ such that $G_{j} \cap G_{j, n_{j}} \neq \boldsymbol{\varnothing}$. Hence the set $\bigcup_{j=1}^{\infty} G_{j, n}$ is dense in $K(x, r)$ and from the fact that $\left\{G_{j}: j=k, k+1, \ldots\right\}$ is also a basis it follows that $\bigcup_{j=k}^{\infty} G_{j, n_{j}}$ is dense (and residual) in $K(x, r)$ for every $k \in N$. So $\limsup C_{j, n_{j}}$ is a residual set in $K(x, r)$ and $\limsup C_{j, n} \notin \mathscr{I}$. This ends the proof.

The assumption of $\mathscr{P}$-measurability of functions $\left\{f_{m, n}\right\}_{m, n \in N}$ is obviously essential in Theorem 3. It is possible to construct a double sequence of functions $\left\{f_{m, n}\right\}_{m, n \in N}$ defined on $[0,1]$ such that for every $m_{k} \underset{k \rightarrow \infty}{\rightarrow} \infty, n_{k} \underset{k \rightarrow \infty}{\rightarrow} \infty \lim _{k \rightarrow \infty} f_{m_{k}, n_{k}}(x)=0$ except on a denumerable set (depending on $\left\{m_{k}\right\}_{k \in N},\left\{n_{k}\right\}_{k \in N}$, but $\lim _{n, m \rightarrow \infty} f_{m, n}(x)$ is not equal to zero at any point of the interval $[0,1]$ (see [4]).

The countable chain condition is also essential in Theorem 3. In [4] there is an example showing that for $X=[0,1], \mathscr{S}-a \sigma$-field of Lebesgue measurable sets and $\mathscr{I}-a \sigma$-ideal of denumerable sets the convergence of every $\left\{f_{m_{k}, m_{k}}\right\}_{k \in N}$ for $\left\{m_{k}\right\}_{k \in N},\left\{n_{k}\right\}_{k \in N}$ tending to infinity does not imply the convergence of $\left\{f_{m, n}\right\}_{m, n \in N}$ except on a denumerable set. It is worth observing that the example is good also if $\mathscr{S}$ is a $\sigma$-field of sets having the Baire property:

In the above considerations (in this part) the behaviour of the pairs ( $\sigma$-field of measurable sets in a $\sigma$-finite measure space, $\sigma$-ideal of null sets) and ( $\sigma$-field of Baire sets, $\sigma$-ideal of sets of the first category) was similar. However, if we assume that the sequence $\left\{m_{k}\right\}_{k \in N}$ and $\left\{n_{k}\right\}_{k \in N}$ are increasing, the situation is different. Sierpiński in [4] has proved that in this case the convergence a.e. of sequences $\left\{f_{m_{k}, n_{k}}\right\}_{k \in N}$ does not imply the convergence a.e. of the sequence $\left\{f_{m, n}\right\}_{m, n \in N}$ even if all functions $f_{m, n}$ are continuous.

Suppose again that $X$ is a complete and separable metric space, $\mathscr{S}-a \sigma$-field of sets having the Baire property and $\mathscr{I}-a \sigma$-ideal of sets of the first category. Then the following theorem holds.

THEOREM 5. If $\left\{f_{m, n}\right\}_{m, n \in N}$ is a double sequence of $\mathscr{\mathscr { S }}$-measurable functions fulfilling the following condition: for all increasing sequences $\left\{m_{k}\right\}_{k \in N}$ and $\left\{n_{k}\right\}_{k \in N}$ of natural numbers $\lim _{k \rightarrow \infty} f_{m_{k}, m_{k}}(x)=0$ I-a.e, on $X$, then $\lim _{m \rightarrow \infty} f_{m, n}(\lambda)=0$ I-a.e. on $X$.

Proof. Suppose that the set $E=\left\{x: \sim\left(\lim _{m, n \rightarrow \infty} f_{m, n}(x)=0\right)\right\}$ does not belong to $\mathscr{I}$. We have

$$
E=\bigcup_{p=1}^{\infty} \bigcap_{M=1}^{\infty} \bigcup_{m=M+1}^{\infty} \bigcup_{n=M+1}^{\infty}\left\{x:\left|f_{m, n}(x)\right|>1 / p\right\},
$$


and so there exists a natural number $p_{0}$ such that the set

$$
D=\bigcap_{M=1}^{\infty} \bigcup_{m=M+1}^{\infty} \bigcup_{n=M+1}^{\infty}\left\{x:\left|f_{m, n}(x)\right|>1 / p_{0}\right\}
$$

is of the second category. $D$ is obviously a set having the Baire property, and so $D=G \Delta P$, where $G$ is open and non-empty and $P$ is of the first category. Let $K(x, r)$ be an open ball with the centre $x$ and the radius $r>0$ included in $G$.

Put $H_{m, n}=\left\{x:\left|f_{m, n}(x)\right|>1 / p_{0}\right\}$ for $m, n \in N$. These sets have the Baire property, and so there exist open sets $\left\{G_{m, n}\right\}_{m, n \in N}$ and the sets of the first category $\left\{P_{m, n}\right\}_{m, n \in N}$ such that $H_{m, n}=G_{m, n} \Delta P_{m, n}$ for every $m, n \in N$.

It is not difficult to prove that for every natural $M$ the set $\bigcup_{m=M+1}^{\infty} \bigcup_{n=M+1}^{\infty} G_{m, n}$ is dense in $K(x, r)$.

Let $\left\{G_{k}: k \in N\right\}$ be a basis for a subspace topology in $K(x, r)$ such that $\left\{G_{k}: k=j, j+1, \ldots\right\}$ is also a basis for every $j \in N$.

The set $\bigcup_{m=1}^{\infty} \bigcup_{n=1}^{\infty} G_{m, n}$ is dense in $K(x, r)$, and so there exist natural numbers $m_{1}$ and $n_{1}$ such that $G_{1} \cap G_{m_{1}, n_{1}} \neq \varnothing$. The set $\bigcup_{m=\max \left(m_{1}, n_{1}\right)+1}^{\infty} \bigcup_{n=\max \left(m_{1}, n_{1}\right)+1}^{\infty} G_{m, n}$ is also dense in $K(x, r)$, and so there exist natural numbers $m_{2}>m_{1}$ and $n_{2}>n_{1}$ such that $G_{2} \cap G_{m_{2}, n_{2}} \neq \varnothing$. Proceeding in this way, we obtain two increasing sequences $\left\{m_{k}\right\}_{k \in N}$ and $\left\{n_{k}\right\}_{k \in N}$ of natural numbers such that $G_{k} \cap G_{m_{k} . n_{k}} \neq \varnothing$ for every $k \in N$. Hence the set $\bigcup_{k=1}^{\infty} G_{m_{k}, n_{k}}$ is dense in $K(x, r)$ and, moreover, for every $j \in N$, the set $\bigcup_{k=j}^{\infty} G_{m_{k}, m_{k}}$ is also dense in $K(x, r)$. So, for every $j \in N$, the set $\bigcup_{k=j}^{\infty} G_{m_{k}, n_{k}}$ is residual in $K(x, r)$ and from the fact that $\bigcup_{k=j}^{\infty} H_{m_{k}, n_{k}} \supset \bigcup_{k=j}^{\infty} G_{m_{k}, n_{k}}-\bigcup_{k=j}^{\infty} P_{m_{k}, n_{k}}$ it follows that $\underset{k}{\limsup } H_{m_{k}, n_{k}}$ is residual in $K(x, r)$. Hence $\underset{k}{\limsup } H_{m_{k}, m_{k}} \notin \mathscr{T}$. But if $x \in \underset{k}{\limsup } H_{m_{k}, m_{k}}$, $\stackrel{k}{k} \lim _{k \rightarrow \infty} f_{m_{k}, m_{k}}(x)$ is not equal to zero - a contradiction. This ends the proof.

\section{References}

[1] R. Engelking, Topologia Ogólna, Warszawa 1976.

[2] C. Goff ma $\mathrm{n}$ and D. Waterman, On upper and lower limits in measure, Fund. Math. 58 (1960), pp. 127-133.

[3] C. Goffman and R. E. Zink, Concerning the measurable boundaries of a real function, ibidem, pp. 105-111.

[4] W. Sierpiński, Sur les suites doubles de fonctions, Fund. Math. 37 (1950), pp. 55-62.

[5] R. Sikorski, Boolean Algebras, New York 1969.

[6] - Funkcje Rzeczywiste, Warszawa 1958.

\section{Circularity of graphs and continua: topology}

\section{by}

\section{Harold Bell, Ezra Brown, R. F. Dickman, Jr.,} and E. L. Green *, (Blacksburg, Va.)

Abstract. A chain in a space $X$ is a finite collection $\left\{K_{1}, \ldots, K_{n}\right\}$ of distinct closed and connected sets such that $K_{i} \cap K_{j} \neq \varnothing$ if and only if $|i-j| \leqslant 1$. A circular chain in $X$ is a collection $\pi$ such that for any $K \in \mathcal{K}, \mathcal{K}-\{K\}$ is a chain. For any locally connected, connected space $X, m(X)$, the circularity of $X$ is defined by

$m(X)=\sup \{n: X$ can be represented as a union of a

circular chain with exactly $n$ elements\}.

The circularity, $\sigma(G)$, of a finite connected graph $G$ is defined by

$\sigma(G)=\sup \{n: G$ can be represented as the union of a circular chain $\pi$ in $G$ such that every member of $\pi$ contains at least one vertex of $G$ \}.

The principal results in this paper are: (1) if $G$ is a (planar) graph, then $G$ can be embedded in a (planar) Peano continuum $X$ with $\sigma(G)=m(X)$. (2) If $X$ is a planar Peano continuum, then $m(X)$ is infinite or even. (3) If $G$ contains a cycle, then $\sigma(G) \geqslant 6$ and if $G$ is planar, then $\sigma(G)$ is even. (4) The $G$ is one of the Kuratowski non-planar graphs, then $\sigma(G)=6$.

In another paper, Circularity of Graphs and continua: Combinatorics, the authors develope combinatorial techniques for the evaluation of the circularity of graphs and show that for any integer $k \geqslant 6$, there exists a non-planar graph $G_{k}$ with $\sigma\left(G_{k}\right)=k$.

1. Introduction. Throughout this paper $X$ will denote a locally connected, connected normal space. For $A \subset X, b_{0}(A)$ denotes the number of components of $A$ less one (or $\infty$ if this number is infinite). The degree of multicoherence, $r(X)$, of $X$ is defined by

(*) $\quad r(X)=\sup \left\{b_{0}(H \cap K): X=H \cup K\right.$ and $H$ and $K$ are closed and connected subsets of $X$ \}.

If $r(X)=0, X$ is said to be unicoherent and we say that $X$ is multicoherent otherwise. $X$ is said to be finitely multicoherent if $0<r(X)<\infty$. If this value is never attained, i.e. $b_{0}(H \cap K)<\infty$ for representations $X=H \cup K$ as in (*), $X$ is said to be weakly-finitely multicoherent. A. H. Stone has studied multicoherent spaces extensively $[6,7,8,9]$ and many authors have studied unicoherent spaces. Stone has raised several interesting questions concerning multicoherent spaces.

- The last author was partially supported by a grant from the National Science foundation. 